\title{
The Light-Quark Magnetic Moment of the Lambda(1405) Antikaon-Nucleon Molecule
}

\author{
Jonathan M. M. Hall ${ }^{\star a}$, Waseem Kamleh ${ }^{a}$, Derek B. Leinweber ${ }^{a}$, Benjamin J. \\ Menadue $^{a, b}$, Benjamin J. Owen ${ }^{a}$, Anthony W. Thomas ${ }^{a, c}$ \\ ${ }^{a}$ Special Research Centre for the Subatomic Structure of Matter (CSSM), \\ Department of Physics, University of Adelaide, Adelaide, South Australia 5005, Australia \\ ${ }^{b}$ National Computational Infrastructure (NCI), \\ Australian National University, Canberra, Australian Capital Territory 0200, Australia \\ ${ }^{c}$ ARC Centre of Excellence for Particle Physics at the Terascale (CoEPP), \\ Department of Physics, University of Adelaide, Adelaide, South Australia 5005, Australia \\ E-mail: jonathan.halleadelaide.edu.au
}

\begin{abstract}
The light-quark sector of the $\Lambda(1405)$ baryon is examined in the context of the recent discovery of a dominant antikaon-nucleon composition at low quark masses. Further evidence for this interpretation of the $\Lambda(1405)$ is presented, by calculating the $u$ and $d$ quark contributions to the $\Lambda(1405)$ magnetic form factors in lattice QCD. The extent to which these quantities are consistent with the exotic molecular description can then be quantified by comparing the results with the equivalent nucleon form factors. Drawing on a recent extension of the graded-symmetry approach for the flavor-singlet components of the $\Lambda(1405)$, the separation of the connected and disconnected contributions is performed in both the flavor-octet and singlet representations. In both cases, the disconnected loop contributions are found to be unexpectedly large. The relationship between the light-quark contributions to the $\Lambda(1405)$ magnetic form factor and the connected contributions of the nucleon magnetic form factors is thus confirmed in the case of lattice QCD, establishing compelling evidence for a $\bar{K} N$ molecular structure of the $\Lambda(1405)$ near the physical point.
\end{abstract}

The 26th International Nuclear Physics Conference

11-16 September, 2016

Adelaide, Australia

${ }^{*}$ Speaker. 


\section{Introduction}

Efforts to understand the internal structure of the $\Lambda(1405)$ baryon has presented a challenge to the scientific community for over 50 years $[1,2]$. While a simple quark-model picture suggests a negative-parity excited state comprising up, down and strange quarks should be heavier than the corresponding negative-parity state of the nucleon, its unexpectedly light mass has prompted a wide range of studies that strongly indicate the presence of a significant contribution from a $\bar{K} N$ bound state [3-11]. This molecular picture of the structure of the $\Lambda(1405)$ has been shown to be prevalent at light quark masses $[8,9]$, while lattice QCD continues to find compelling evidence for the constituent-quark model of the $\Lambda(1405)$ at both intermediate and heavy quark masses [10, 12]. Describing the behaviour of the $\Lambda(1405)$ over a wide range of quark masses has recently been shown to require a composite picture, whereby a three-quark state with non-zero bare mass encounters strong interactions with nearby multi-hadron states, particularly $\bar{K} N$ and $\pi \Sigma$, which contribute significantly to the properties of the $\Lambda(1405)$ near the physical point $[10,13,14]$. This development serves to bridge the constituent-quark picture at heavy quark masses and the molecular $\bar{K} N$ dominance of the $\Lambda(1405)$ at light quark masses.

While lattice QCD simulation methods have demonstrated unprecedented accuracy in determining baryon ground state observables at relatively low quark masses $[15,16]$, advancement in excited-baryon form factors is still at an early stage [10,17-20]. Nevertheless, calculations of a vanishing strange magnetic form factor for the $\Lambda(1405)$ at small quark masses are crucial in uncovering how the $\bar{K} N$ molecular behaviour appears on the lattice [10]. Since the $s$ quark present in the $\bar{K} N$ system is confined within the spin-0 kaon in a relative $S$ wave with respect to a nucleon, the angular momentum must be zero. Hence, the strange quark cannot contribute to the magnetic form factor of a $\Lambda(1405)$ composed as a molecular $\bar{K} N$ bound state.

In the case of the light-quark sector of the $\Lambda(1405)$, lattice results must omit photon couplings to the disconnected quark-antiquark loops, due to computational intensiveness and difficulty in isolating a signal for excited states [10,13,21-27]. Diagrams corresponding to the included and omitted contributions to the form factors for the process $\Lambda^{*} \rightarrow K^{-} p$, for example, are shown in Fig. 1. (The star superscript notation indicates that the resonance has odd parity). Thus, careful accounting of the omitted contributions are vital in a meaningful comparison of the form factors of the $\Lambda(1405)$ compared to those of the nucleon, and a reliable estimate of the magnitude of these partial quenching effects must be made [28].

\section{Loop contributions to the magnetic form factor}

In the $\bar{K} N$ picture, the spin- 0 kaon is in a relative $S$ wave about the nucleon, and thus, in the absence of orbital angular momentum, the light-quark contributions to the magnetic form factor of the $\Lambda(1405)$ can be attributed solely to the nucleon. Since couplings derived from SU(3) symmetry for $\Lambda^{*} \rightarrow K^{-} p$ and $\Lambda^{*} \rightarrow \bar{K}^{0} n$ are equal, the light sector contribution may be written as an average of $n$ and $p$ magnetic form factors

$$
\left|\Lambda^{*}\right\rangle=\frac{1}{\sqrt{2}}\left(\left|K^{-} p\right\rangle+\left|\bar{K}^{0} n\right\rangle\right) .
$$



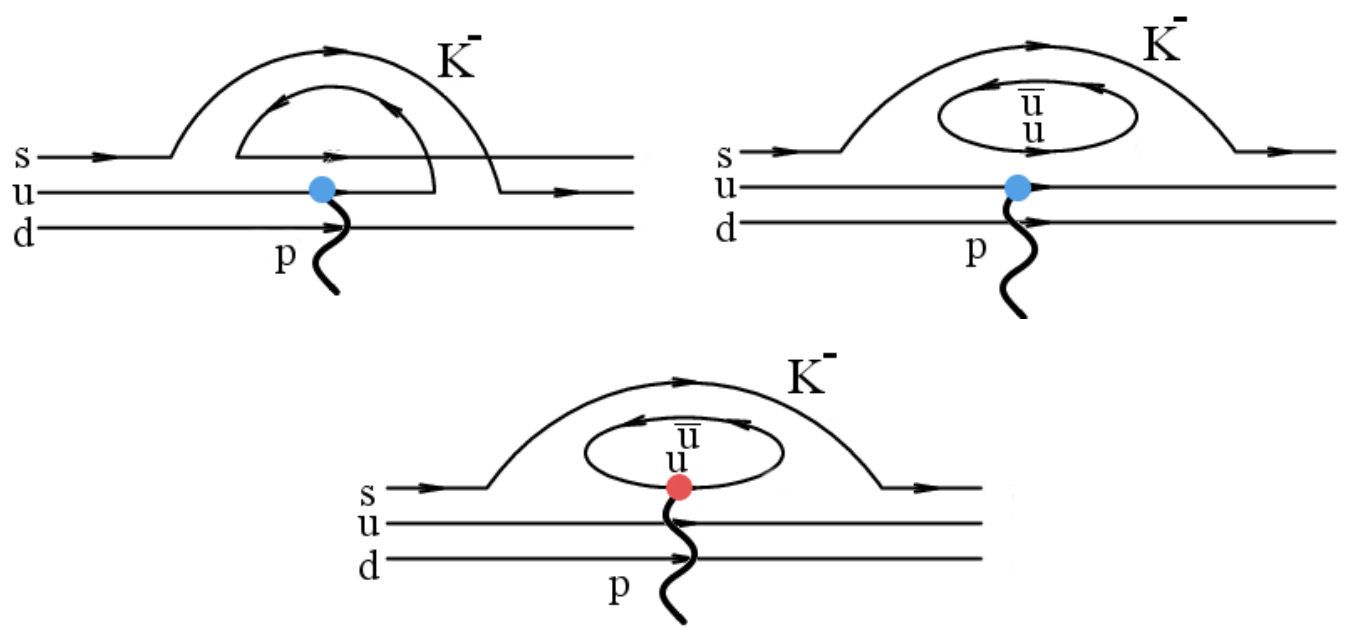

Figure 1: (color online). The quark flow diagrams for the process $\Lambda(1405) \rightarrow K^{-} p$ can be decomposed into a completely-connected part and two parts involving disconnected sea-quark loop contributions. The upperleft completely-connected diagram and the upper-right diagram (blue dots) are included in the lattice QCD calculations as the photon interacts with a valence quark. The case where a photon couples to a disconnected sea quark loop, illustrated in the lower diagram (red dot), is not included in the lattice QCD calculations.

In full QCD, which includes the disconnected sea-quark loop contributions, the form of the lightquark magnetic form factor takes the form

$$
\begin{aligned}
\left\langle\Lambda^{*}\left|\hat{\mu}_{q}\right| \Lambda^{*}\right\rangle & =\frac{1}{2}\left\langle K^{-} p\left|\hat{\mu}_{q}\right| K^{-} p\right\rangle+\frac{1}{2}\left\langle\bar{K}^{0} n\left|\hat{\mu}_{q}\right| \bar{K}^{0} n\right\rangle, \\
& =\frac{1}{2}\left\langle p\left|\hat{\mu}_{q}\right| p\right\rangle+\frac{1}{2}\left\langle n\left|\hat{\mu}_{q}\right| n\right\rangle .
\end{aligned}
$$

While the $\Lambda(1405)$ is identified as the low-lying flavor-singlet baryon in the $S U$ (3)-flavor limit, as one approaches the physical regime, significant mixing with octet-flavor symmetry is encountered [12]. Therefore one needs to consider both flavor-octet and flavor-singlet couplings for $\Lambda^{*} \rightarrow \bar{K} N$. In addressing the latter, we draw upon the recently developed graded-symmetry approach for singlet baryons [29], which augments the standard octet-baryon Lagrangian with the necessary additional terms.

First, consider the contributions to the singlet component of the $\Lambda(1405)$, denoted $\Lambda^{\prime *}$, where the prime indicates that a singlet representation is taken. In the case of the process $\Lambda^{*} \rightarrow K^{-} p$, the relevant ghost term in the Lagrangian takes the form [29]

$$
-g_{s} \sqrt{\frac{2}{3}} \bar{\Lambda}^{* *} \tilde{K}^{-} \tilde{\Lambda}_{p, \tilde{u}}^{+}
$$

where $g_{s}$ is taken to be the coupling of the singlet to octet-octet process $\Lambda^{\prime *} \rightarrow \pi_{0} \Sigma_{0}$. Here, the notation of Ref. [29] is used. $\tilde{K}^{-}$is composed of a strange quark (s) and a ghost anti-up quark $(\overline{\tilde{u}})$ and $\tilde{\Lambda}_{p, \tilde{u}}^{+}$represents a proton-like particle composed of $\tilde{u} u d$, with the normal quarks in an antisymmetric formation. The factor $\sqrt{2 / 3}$ is derived from the $S U(3 \mid 3)$ symmetry relations that govern the augmented Lagrangian. Recalling the full QCD vertex required for this process,

$$
g_{s} \bar{\Lambda}^{*} K^{-} p
$$


the relative sizes of the connected and disconnected loop contributions can be resolved- the connected diagram has weight $(1 / 3) g_{s}^{2}$ and the disconnected diagram has weight $(2 / 3) g_{s}^{2}$. The same ratio is found in the case of $\Lambda^{* *} \rightarrow \bar{K}^{0} n$, where a $d$ quark participates in the loop in full QCD.

Since flavor-symmetry breaking leads to significant singlet and octet mixing in the flavor components of the $\Lambda(1405)$, particularly near the physical point [12], one must also consider the octetto-octet meson and baryon contributions. Upon partial quenching, the corresponding couplings lead to the same ratio of $\sqrt{2 / 3}$. Thus the connected diagram holds a weight of $1 / 3$ and the disconnected diagram holds a weight of $2 / 3$ of the full QCD process independent of the representation of the $\Lambda(1405)$. As a result, the calculation of the partially quenched value of the magnetic form factor is straightforward. For example, because half of the $u$-quark contributions are in the disconnected loop, one can obtain the $u$-quark contributions to the proton, $u_{p}$, by subtracting off $1 / 2 \times 2 / 3=1 / 3$ of the full QCD contribution. The $u$-quark contribution to the neutron, $u_{n}$, on the other hand, is fully included in the lattice QCD calculation, since the disconnected quark-loop flavor is a $d$ quark, not a $u$ quark, so no adjustment is required

$$
\begin{aligned}
& \left\langle\Lambda^{*}\left|\hat{\mu}_{u}^{\mathrm{conn}}\right| \Lambda^{*}\right\rangle= \\
& \frac{1}{2}\left(\left\langle K^{-} p\left|\hat{\mu}_{u}\right| K^{-} p\right\rangle-\frac{1}{2} \frac{2}{3}\left\langle K^{-} p\left|\hat{\mu}_{u}\right| K^{-} p\right\rangle\right)+\frac{1}{2}\left\langle\bar{K}^{0} n\left|\hat{\mu}_{u}\right| \bar{K}^{0} n\right\rangle=\frac{1}{2}\left(2 u_{p}-\frac{2}{3} u_{p}+u_{n}\right) .
\end{aligned}
$$

The first two terms of Eq. (2.5) represent the connected $u$-quark contribution from the proton component within the $\Lambda(1405)$. The first of these terms provides the full QCD contribution while the second term subtracts half of the weight of the disconnected sea-quark loop associated with photon couplings to the disconnected loop. Similarly, for the $d$-quark contribution, a magnetic form factor of $\frac{1}{2}\left(2 d_{n}-\frac{2}{3} d_{n}+d_{p}\right)$ is obtained. Note that, under charge symmetry, the two light quark contributions, $\hat{\mu}_{\ell}^{\text {conn }}$ become equal for quarks of unit charge

$$
\left\langle\Lambda^{*}\left|\hat{\mu}_{\ell}^{\text {conn }}\right| \Lambda^{*}\right\rangle=\frac{1}{2}\left(2 u_{p}-\frac{2}{3} u_{p}+u_{n}\right) .
$$

\section{Lattice QCD results}

To test the $\bar{K} N$ model prediction of Eq. (2.6), the same set of configurations explored in Ref. [10] are used, leading to a calculation of the left-hand side of the equation, $\left\langle\Lambda^{*}\left|\hat{\mu}_{\ell}^{\text {conn }}\right| \Lambda^{*}\right\rangle$. The calculation is based on the $32^{3} \times 64$ full-QCD ensembles created by the PACS-CS collaboration [30], made available through the International Lattice Data Grid (ILDG) [31]. The ensembles provide a lattice volume of $(2.9 \mathrm{fm})^{3}$ with five different masses for the light $u$ and $d$ quarks, and constant strange-quark simulation parameters. A valence strange quark with a hopping parameter of $\kappa_{s}=0.13665$ is simulated, reproducing the correct kaon mass in the physical limit [32]. The squared pion mass is chosen as a renormalization group invariant measure of the quark mass, and the scale is set via the Sommer parameter [33] with $r_{0}=0.492 \mathrm{fm} \mathrm{[30].}$

In calculating the right-hand side of Eq. (2.6), the nucleon magnetic form factors are determined on these lattices using the methods introduced in Ref. [34, 35], providing values of $u_{p}=1.216(17) \mu_{N}$ and $u_{n}=-0.366(19) \mu_{N}$ at the lightest pion mass, and the lowest nontrivial momentum transfer of $Q^{2} \simeq 0.16 \mathrm{GeV}^{2} / c^{2}$. Figure 2 shows the results for the light- and strange-quark 


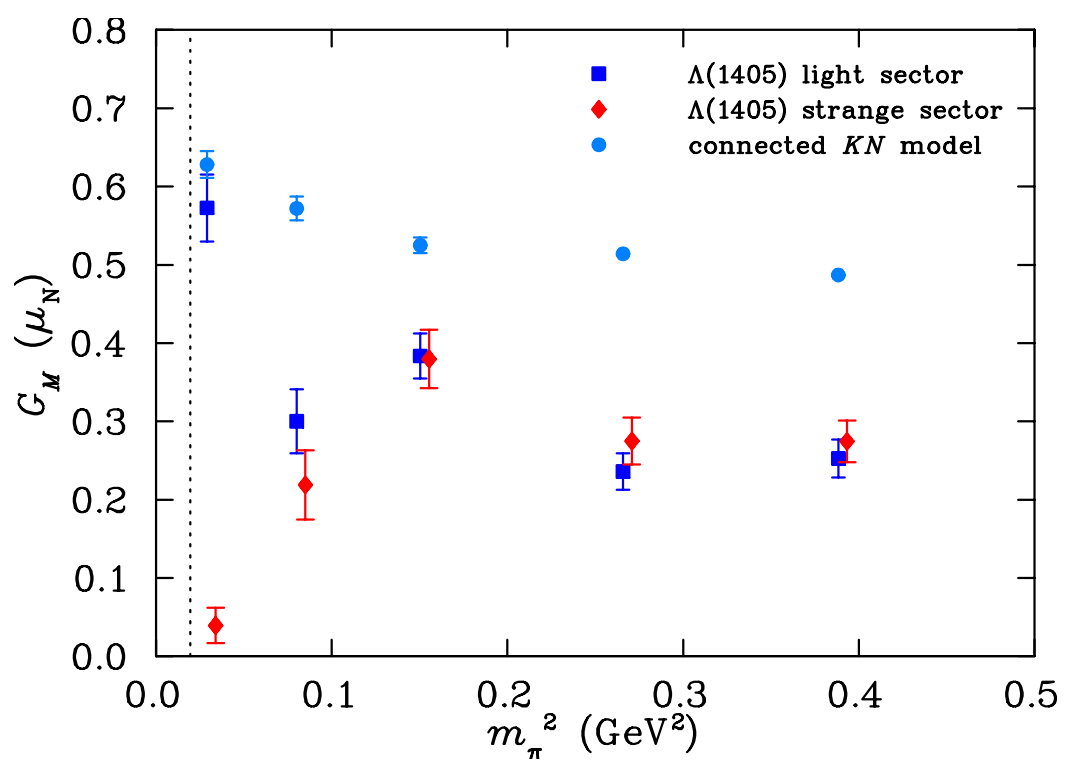

Figure 2: The light $\left(u\right.$ or $d$ ) and strange quark contributions to the magnetic form factor of the $\Lambda(1405)$ at $Q^{2} \simeq 0.16$ $\mathrm{GeV}^{2} / c^{2}$ from Ref. [10] are presented as a function of the light $u$ - and $d$-quark masses, indicated by the squared pion mass, $m_{\pi}^{2}$. Sector contributions are for single quarks of unit charge. The lattice calculations are compared to the predictions of the connected $\bar{K} N$ model developed herein. The vertical dashed line indicates the physical pion mass. The strange form factor results are offset a small amount from the light sector in the $m_{\pi}^{2}$-axis for clarity.

magnetic form factors of the $\Lambda(1405)$, plotted as a function of pion mass. The flavor symmetry present at heavy quark masses is broken as the $u$ and $d$ masses approach the physical point, where the strange magnetic form factor drops nearly to zero. The light quark sector contribution differs significantly from the molecular $\bar{K} N$ model prediction until the lightest quark mass is reached. The direct matrix element calculation, $\left\langle\Lambda^{*}\left|\hat{\mu}_{\ell}^{\text {conn }}\right| \Lambda^{*}\right\rangle$ agrees with the prediction of the connected $\bar{K} N$ model, shown in Eq. (2.6). At the lightest pion mass, the light-quark magnetic form factor of the $\Lambda(1405)$ is [10]

$$
\left\langle\Lambda^{*}\left|\hat{\mu}_{\ell}^{\operatorname{conn}}\left(Q^{2}\right)\right| \Lambda^{*}\right\rangle=0.58(5) \mu_{N},
$$

at $Q^{2} \simeq 0.16 \mathrm{GeV}^{2} / c^{2}$. The connected $\bar{K} N$ model of Eq. (2.6) predicts

$$
\left\langle\Lambda^{*}\left|\hat{\mu}_{\ell}^{\text {conn }}\left(Q^{2}\right)\right| \Lambda^{*}\right\rangle=0.63(2) \mu_{N} .
$$

This agreement confirms that the $\Lambda(1405)$ observed in lattice QCD is dominated by a molecular $\bar{K} N$ structure near the physical point.

Note that there is a significant shift in the prediction due to the omission of the photon couplings to the disconnected sea-quark loop. In the case where such couplings are included, the prediction of the $\bar{K} N$ model is much larger, $\left\langle\Lambda^{*}\left|\hat{\mu}_{\ell}\right| \Lambda^{*}\right\rangle=\left(2 u_{p}+u_{n}\right) / 2=1.03(2) \mu_{N}$. Thus, a determination of these disconnected-loop contributions directly from lattice QCD would be of great value as a next step forward, particularly for resonances where coupled-channel dynamics play an important role. 


\section{Conclusion}

The light-quark sector contributions to the magnetic form factor of the $\Lambda(1405)$, calculated in lattice QCD, have been compared to the predictions of a molecular $\bar{K} N$ model. By identifying the quark-flow connected contributions to the magnetic form factors of the $\Lambda(1405)$, a quantitative analysis of light-quark contributions to the form factors shows that they are consistent with a molecular bound-state description near the physical point.

Identification and removal of the quark-flow disconnected contributions to the $\bar{K} N$ model were conducted using a recently extended graded-symmetry approach [29], which includes couplings to the flavor-singlet components of baryons. It is found that the ratio of connected to disconnected contributions is identical for both flavor-singlet and flavor-octet representations of the $\Lambda(1405)$.

Using new results for the magnetic form factors of the nucleon at a light pion mass of $m_{\pi}=156$ $\mathrm{MeV}$ [28], the connected $\bar{K} N$ model predicts a light-quark sector contribution to the $\Lambda(1405)$ of $0.63(2) \mu_{N}$, which is consistent with the direct calculation of $0.58(5) \mu_{N}$ from Ref. [10]. This investigation thus provides further evidence that the internal structure of the $\Lambda(1405)$ is dominated by a $\bar{K} N$ molecule.

\section{Acknowledgments}

We thank the PACS-CS Collaboration for making their $2+1$ flavor configurations available and the ongoing support of the ILDG. This research was undertaken with the assistance of the University of Adelaide's Phoenix cluster and resources at the NCI National Facility in Canberra, Australia. NCI resources were provided through the National Computational Merit Allocation Scheme, supported by the Australian Government and the University of Adelaide Partner Share. This research is supported by the Australian Research Council through the ARC Centre of Excellence for Particle Physics at the Terascale (CE110001104), and through Grants No. LE160100051, DP151103101 (A.W.T.), DP150103164, DP120104627 and LE120100181 (D.B.L.).

\section{References}

[1] R. Dalitz and S. Tuan, Annals Phys. 10, 307 (1960).

[2] R. Dalitz, T. Wong and G. Rajasekaran, Phys. Rev. 153, 1617 (1967).

[3] E. Veit, B. K. Jennings, R. Barrett and A. W. Thomas, Phys. Lett. B137, 415 (1984).

[4] N. Kaiser, P. Siegel and W. Weise, Nucl. Phys. A594, 325 (1995).

[5] E. Oset and A. Ramos, Nucl. Phys. A 635, 99 (1998).

[6] L. S. Geng, E. Oset and M. Doring, Eur. Phys. J. A32, 201 (2007).

[7] Z.-H. Guo and J. A. Oller, Phys. Rev. C 87, 035202 (2013).

[8] R. Molina and M. Döring, Phys. Rev. D94, 056010 (2016).

[9] J. Oller, Int. J. Mod. Phys. Conf. Ser. 26, 1460096 (2014).

[10] J. M. M. Hall et al., Phys. Rev. Lett. 114, 132002 (2015).

[11] J. M. M. Hall et al., PoS LATTICE2014, 094 (2014). 
[12] B. J. Menadue, W. Kamleh, D. B. Leinweber and M. S. Mahbub, Phys. Rev. Lett. 108, 112001 (2012).

[13] J. Hall, A. C. P. Hsu, D. Leinweber, A. Thomas and R. Young, Phys. Rev. D87, 094510 (2013).

[14] Z.-W. Liu, J. M. M. Hall, D. B. Leinweber, A. W. Thomas and J.-J. Wu, Phys. Rev. D 95, 014506 (2017).

[15] J. Green et al., Phys. Rev. D92, 031501 (2015).

[16] $\chi$ QCD Collaboration, R. S. Sufian et al., Phys. Rev. Lett. 118, 042001 (2017).

[17] B. J. Owen, W. Kamleh, D. B. Leinweber, M. S. Mahbub and B. J. Menadue, PoS LATTICE2013, 277 (2014).

[18] D. S. Roberts, W. Kamleh and D. B. Leinweber, Phys. Rev. D89, 074501 (2014).

[19] B. Owen, W. Kamleh, D. Leinweber, S. Mahbub and B. Menadue, PoS LATTICE2014, 159 (2014).

[20] F. M. Stokes et al., Phys. Rev. D92, 114506 (2015).

[21] M. S. Mahbub, W. Kamleh, D. B. Leinweber, P. J. Moran and A. G. Williams, Phys. Rev. D87, 094506 (2013).

[22] C. Morningstar et al., Phys. Rev. D88, 014511 (2013).

[23] C. Alexandrou, T. Leontiou, C. N. Papanicolas and E. Stiliaris, Phys. Rev. D91, 014506 (2015).

[24] B. J. Owen, W. Kamleh, D. B. Leinweber, M. S. Mahbub and B. J. Menadue, Phys. Rev. D92, 034513 (2015).

[25] D. Leinweber et al., JPS Conf. Proc. 10, 010011 (2016).

[26] Z.-W. Liu et al., 1607.04536.

[27] C. B. Lang, L. Leskovec, M. Padmanath and S. Prelovsek, Phys. Rev. D 95, 014510 (2017).

[28] J. M. M. Hall et al., 1612.07477.

[29] J. M. M. Hall and D. B. Leinweber, Phys. Rev. D94, 094004 (2016).

[30] PACS-CS Collaboration, S. Aoki et al., Phys. Rev. D 79, 034503 (2009).

[31] M. G. Beckett et al., Comput. Phys. Commun. 182, 1208 (2011).

[32] B. J. Menadue, W. Kamleh, D. B. Leinweber, M. S. Mahbub and B. J. Owen, PoS LATTICE2012, 178 (2012).

[33] R. Sommer, Nucl. Phys. B411, 839 (1994).

[34] D. B. Leinweber, R. Woloshyn and T. Draper, Phys. Rev. D43, 1659 (1991).

[35] S. Boinepalli, D. Leinweber, A. Williams, J. Zanotti and J. Zhang, Phys. Rev. D74, 093005 (2006). 